\title{
Cusp relations for local strongly decaying properties in electronic systems
}

\author{
Shubin Liu and Robert G. Parr \\ Department of Chemistry, University of North Carolina, Chapel Hill, North Carolina 27599-3290 \\ A. Nagy \\ Institute of Theoretical Physics, Kossuth Lajos University, H-4010 Debrecen, Hungary
}

(Received 24 April 1995)

\begin{abstract}
Several expressions are derived that allow one to evaluate any local property at nuclear cusps in atoms and molecules. Applications of the formulas to the Hartree-Fock density and its derivatives for first- and secondrow atoms show agreement with Kato's cusp condition. Applications to the exact exchange-only and exchangecorrelation energy potential for neutral atoms are given. It is shown that for an atom, the values of $V_{\mathrm{x}}(0)$ and $V_{\mathrm{xc}}(0)$ are both close to the negative of the charge of the nucleus.

PACS number(s): 31.15.- $\mathrm{p}$
\end{abstract}

\section{INTRODUCTION}

There is considerable interest in density-functional theory (DFT) [1] as a tool for calculating properties of electronic systems ranging from atoms and molecules to solids. Working with density $\rho$ instead of wave function $\Psi$, DFT is capable of reducing calculation tremendously because $\rho$ is only a function of three variables while $\Psi$ is a function of $3 N$ variables, where $N$ is the number of electrons in the system of concern. Even in the Kohn-Sham scheme [2], in which the concept of orbital is still retained, it is generally accepted that the results of DFT are at about the Hartree-Fock plus MP2 level even though calculations are performed with a single Slater determinant wave function, just as in HartreeFock theory.

Among the unsolved problems in DFT are the values of various quantities at a nucleus (or cusp). It is hard to obtain an accurate value at this point because in most circumstances expressions to give the value are singular at $\mathbf{r}=\mathbf{0}$. Thus one is unable to approach the point smoothly. However, the value of a quantity at a cusp is an important entity. It can serve either as a criterion to justify a method or an approximation, or as a means to improve some approach involved. For example, Kato's cusp condition [3],

$$
\rho(0)+\frac{\rho^{\prime}(0)}{2 Z}=0
$$

where $\rho$ and $\rho^{\prime}$ are the density and its derivative, respectively, and $Z$ is the nuclear charge, is usually employed in testing an approximate wave function or density in calculation to see if it behaves properly near and at a nucleus. Other examples, showing the importance of a quantity at a nucleus, are the Thomas-Fermi [4,5] and Thomas-Fermi-Dirac [6] models for neutral atoms. It is well known that these models give an electron density that, although normalizable, diverges at an atomic nucleus. But when one incorporates one additional constraint [7], namely,

$$
\int e^{-2 k r} \nabla^{2} \rho(\mathbf{r}) d \mathbf{r}<\infty
$$

to force densities to satisfy the cusp condition, electron densities and total energies are vastly improved. We will show in later sections that the constraint form (2) is similar to part of an expression for the density at a nucleus.

The importance of a value at a nucleus is not restricted only to the density. In DFT, the exchange-correlation potential $V_{\mathrm{xc}}$ has special interest, since in the Kohn-Sham method the only unknown is this term. Much progress of DFT in recent years is related to this quantity [8], for which various approximate forms beyond the local-density-approximation (LDA) or local-spin-density approximation (LSDA) have been obtained and have been found to give an overall improvement to the exchange-correlation energies. Unfortunately, however, in a very recent paper by Umrigar and Gonze [9], it is shown that, although the generalized gradient approximation (GGA) yields improved energies compared to the local-density approximation, the exchange-correlation potentials obtained from the GGA and other familiar approximations are in poor agreement with exact potentials, especially near a nucleus. We shall give in the present paper accurate values of $V_{\mathrm{xc}}$ at the nucleus for the neutral atoms $\mathrm{He}, \mathrm{Be}, \mathrm{Ne}, \mathrm{Mg}$, and $\mathrm{Ar}$.

Although the expression to give the value of a quantity may be singular at the nucleus, in most of the cases we consider here its value at this point is definitely finite. In Sec. II, proof of a number of formulations for this purpose is given based on Green's theorem. One finds that the exact value of any quantity can be obtained at a nuclear cusp in terms of its gradients and Laplacians over the whole range of integration. In Sec. III, application of the formulas is made to obtain densities and their first derivatives. Excellent agreement with Kato's cusp condition is observed. In Sec. IV, we apply the formulas to the exchange-only and exchangecorrelation potentials, and determine data for $V_{\mathrm{x}}(0)$ and $V_{\mathrm{xc}}(0)$ for atoms. Discussions are presented in Sec. $\mathrm{V}$ to model the data for $V_{\mathrm{x}}(0)$ and $V_{\mathrm{xc}}(0)$. Final remarks are included in Sec. VI. Atomic units are used throughout.

\section{THE BASIC CUSP RELATIONS}

Theorem. For any quantity $F(\mathbf{r})$ in an electronic system such that $F(\mathbf{r})$ falls off faster that $1 / r$ and its gradient falls 
off faster than $1 / r^{2}$, the value at a nucleus (cusp) can be evaluated from any one of the following expressions:

$$
\begin{gathered}
F(0)=-\frac{1}{4 \pi} \int \frac{\nabla^{2} F(\mathbf{r})}{|\mathbf{r}|} d \mathbf{r} \\
F(0)=-\frac{1}{4 \pi} \int \frac{\mathbf{r} \cdot \nabla F(\mathbf{r})}{r^{3}} d \mathbf{r} \\
F(0)=-\frac{1}{4 \pi} \int \frac{e^{-\alpha r} \nabla^{2} F(\mathbf{r})}{|\mathbf{r}|} d \mathbf{r}+\frac{1}{4 \pi} \int \frac{\alpha^{2} e^{-\alpha r} F(\mathbf{r})}{|\mathbf{r}|} d \mathbf{r}
\end{gathered}
$$

and

$$
F(0)=-\frac{1}{4 \pi} \int \frac{e^{-\beta r} \mathbf{r} \cdot \nabla F(\mathbf{r})}{r^{3}} d \mathbf{r}+\frac{1}{4 \pi} \int \frac{\beta e^{-\beta r} F(\mathbf{r})}{r^{2}} d \mathbf{r},
$$

where $\alpha$ and $\beta$ are arbitrary constants. Falloff of $F$ faster than $1 / r$ means that $|F(\mathbf{r})|<1 / r$ for large enough $r$.

Proof of Equations. We use the two identities of Green's theorem [10]

$$
\int_{V \rightarrow \infty}\left[\phi \nabla^{2} \psi+\nabla \phi \cdot \nabla \psi\right] d V=\lim _{r \rightarrow \infty} \oiint_{S_{r}} \phi \nabla \psi \cdot d \mathbf{a},
$$

and

$$
\int_{V \rightarrow \infty}\left(\phi \nabla^{2} \psi-\psi \nabla^{2} \phi\right) d V=\lim _{r \rightarrow \infty} \oiint_{s_{r}}[\phi \nabla \psi-\psi \nabla \phi] \cdot d \mathbf{a} .
$$

(i) Choose $\phi=F(\mathbf{r})$ and $\psi=1 /|\mathbf{r}|$ and insert them into Eq. (7). Because $F(\mathbf{r})$ decays faster than $1 / r$, the right-hand side of Eq. (7) is zero:

$\lim _{r \rightarrow \infty} \oiint_{S_{r}} F(\mathbf{r}) \boldsymbol{\nabla} \frac{1}{|\mathbf{r}|} \cdot d \mathbf{a}<\lim _{r \rightarrow \infty} \oiint_{S_{r}} \frac{1}{r} \frac{\mathbf{r}}{r^{3}} \cdot \hat{\mathbf{r}} r^{2} d \Omega=0$.

In the left side of Eq. (7) we use

$$
\nabla^{2} \frac{1}{|\mathbf{r}|}=-4 \pi \delta(\mathbf{r})
$$

and

$$
\int F(\mathbf{r}) \delta(\mathbf{r}) d \mathbf{r}=F(0)
$$

One then obtains Eq. (4).

(ii) Choose $\phi=F(\mathbf{r})$ and $\nabla \psi=e^{-\alpha r} \nabla 1 /|\mathbf{r}|$, where $\alpha$ is an arbitrary parameter, and inserting them also into Eq. (7), straightforwardly one obtains Eq. (6).

(iii) Choose $\psi=1 /|\mathbf{r}|$ and $\phi=F(\mathbf{r})$ and insert them into Eq. (8). Besides Eq. (9), since $\nabla F(\mathbf{r})$ decays faster than $1 / r^{2}$

$$
\lim _{r \rightarrow \infty} \oiint_{s_{r}} \frac{1}{|\mathbf{r}|} \nabla F(\mathbf{r}) \cdot d \mathbf{a}<\lim _{r \rightarrow \infty} \oiint_{s_{r}} \frac{\hat{\mathbf{r}}}{r^{3}} \cdot \hat{\mathbf{r}} r^{2} d \Omega=0 .
$$

Thus the right-hand side of Eq. (8) is zero. Upon using Eqs. (10) and (11), Eq. (3) follows.

(iv) Choosing $\psi=e^{-\beta r} /|\mathbf{r}|$ and $\phi=F(\mathbf{r})$, where $\beta$ is an arbitrary parameter, and inserting them into Eq. (8), by similar steps, one has Eq. (5).

Q.E.D.

It is interesting to notice the following. (i) In each of the expressions (5) and (6), an arbitrary constant ( $\alpha$ or $\beta$ ) enters the evaluation of any quantity at the $\mathbf{r}=\mathbf{0}$ point which is supposedly always constant and finite. (ii) The conditions that restrict the theorem, which state that a quantity and its derivative must decay faster than $1 / r$ and $1 / r^{2}$, respectively, are applicable to atomic and molecular systems, in which it is known that quantities, such as wave function, density, and potentials, decay exponentially.

\section{THE ELECTRON DENSITY AND ITS DERIVATIVE AT A NUCLEUS}

Setting $F(\mathbf{r})=\rho(\mathbf{r})$, one obtains four expressions for evaluating the density at a nucleus:

$$
\begin{gathered}
\rho(0)=-\frac{1}{4 \pi} \int \frac{\nabla^{2} \rho(\mathbf{r})}{|\mathbf{r}|} d \mathbf{r} \\
\rho(0)=-\frac{1}{4 \pi} \int \frac{\mathbf{r} \cdot \nabla \rho(\mathbf{r})}{r^{3}} d \mathbf{r} \\
\rho(0)=-\frac{1}{4 \pi} \int \frac{e^{-\alpha r} \nabla^{2} \rho(\mathbf{r})}{|\mathbf{r}|} d \mathbf{r}+\frac{\alpha^{2}}{4 \pi} \int \frac{e^{-\alpha r} \rho(\mathbf{r})}{|\mathbf{r}|} d \mathbf{r} \\
\rho(0)=-\frac{1}{4 \pi} \int \frac{e^{-\beta r} \mathbf{r} \cdot \nabla \rho(\mathbf{r})}{r^{3}} d \mathbf{r}+\frac{\beta}{4 \pi} \int \frac{e^{-\beta r} \rho(\mathbf{r})}{r^{2}} d \mathbf{r}
\end{gathered}
$$

where $\alpha$ and $\beta$ are arbitrary constants. In the spherically symmetrical case, by setting $F(r)=\rho^{\prime}(r)$, one obtains four corresponding expressions for the density derivative at a nucleus:

$$
\begin{gathered}
\rho^{\prime}(0)=-\int\left[\rho^{\prime \prime}(r)+\frac{2}{r} \rho^{\prime}(r)\right] r d r, \\
\rho^{\prime}(0)=-\int \rho^{\prime \prime}(r) d r,
\end{gathered}
$$

$$
\begin{gathered}
\rho^{\prime}(0)=-\int r e^{-\alpha r}\left[\rho^{\prime \prime \prime}(r)+\frac{2}{r} \rho^{\prime \prime}(r)-\alpha^{2} \rho^{\prime}(r)\right] d r, \\
\rho^{\prime}(0)=-\int e^{-\beta r}\left[\rho^{\prime \prime}(r)-\beta \rho^{\prime}(r)\right] d r,
\end{gathered}
$$

where $\alpha$ and $\beta$ are arbitrary constants, and $p^{\prime \prime}(r)$ and $p^{\prime \prime \prime}(r)$ stand for the second- and third-order density derivatives with respect to $r$, respectively. 
TABLE I. Density at the nuclei cusps from Eqs. (13)-(16) for neutral atoms ranging from He to Ar. For comparison, exact values and values using the Hiller-Sucher-Feinberg identity are also shown.

\begin{tabular}{lcccccc}
\hline \hline & \multicolumn{7}{c}{ Density at nucleus } \\
& Formula I & Formula II & Formula III & Formula IV & $\rho_{\text {HSF }}(0)^{b}$ & Exact $^{\mathrm{c}}$ \\
\hline $\mathrm{He}$ & 3.597 & 3.597 & 3.597 & 3.597 & 3.562 & 3.60 \\
$\mathrm{Li}$ & 13.834 & 13.834 & 13.834 & 13.834 & & \\
$\mathrm{Be}$ & 35.428 & 35.428 & 35.428 & 35.428 & 35.311 & \\
$\mathrm{~B}$ & 71.985 & 71.985 & 71.985 & 71.985 & & 127.56 \\
$\mathrm{C}$ & 127.556 & 127.555 & 127.556 & 127.556 & & \\
$\mathrm{~N}$ & 206.135 & 206.135 & 206.135 & 206.135 & & \\
$\mathrm{O}$ & 311.975 & 311.975 & 311.975 & 311.975 & & \\
$\mathrm{~F}$ & 448.710 & 448.710 & 448.710 & 448.710 & & \\
$\mathrm{Ne}$ & 620.146 & 620.146 & 620.146 & 620.146 & 619.13 & \\
$\mathrm{Na}$ & 833.833 & 833.833 & 833.833 & 833.833 & & 1093.73 \\
$\mathrm{Mg}$ & 1093.731 & 1093.731 & 1093.731 & 1093.731 & & \\
$\mathrm{Al}$ & 1402.907 & 1402.906 & 1402.907 & 1402.907 & & \\
$\mathrm{Si}$ & 1765.708 & 1765.707 & 1765.708 & 1765.708 & & \\
$\mathrm{P}$ & 2186.309 & 2186.309 & 2186.309 & 2186.309 & & \\
$\mathrm{~S}$ & 2670.098 & 2670.098 & 2670.098 & 2670.098 & & \\
$\mathrm{Cl}$ & 3218.036 & 3218.036 & 3218.036 & 3218.036 & & \\
$\mathrm{Ar}$ & 3840.223 & 3840.222 & 3840.223 & 3840.223 & & \\
\hline \hline
\end{tabular}

${ }^{\mathrm{a}}$ Formulas I-IV represent Eqs. (13)-(16), respectively.

${ }^{b}$ From Ref. [13].

${ }^{c}$ From Ref. [22].

It is interesting to notice that Parr and Ghosh [7] used Eq. (2), and Zhou and Parr [11] used

$$
\int e^{-k^{\prime} r} \rho^{4 / 3}(\mathbf{r}) d \mathbf{r}<\infty
$$

where $k$ and $k^{\prime}$ are parameters to be determined, as constraints to force the density to satisfy both cusp and asympotic behavior conditions. They found much improvement both in density behavior and in energies. The forms they introduced are much like the numerators of components in the right-hand side of Eq. (15). To see how one can obtain the constraint Eq. (2) from Eq. (15), one takes the derivative of both sides of Eq. (15) with respect to the parameter $\alpha$. Since $\alpha$ is arbitrary, $d \rho(0) / d \alpha=0$. Thus,

$$
\begin{array}{r}
\int e^{-\alpha r} \nabla^{2} \rho(\mathbf{r}) d \mathbf{r}-\alpha^{2} \int e^{-\alpha r} \rho(\mathbf{r}) d \mathbf{r}+2 \alpha \int \frac{e^{-\alpha r} \rho(\mathbf{r})}{4} d \mathbf{r} \\
=0
\end{array}
$$

for any $\alpha$. In this equation the first term on the left side is exactly the constraint of Eq. (2), while the second term is similar to Eq. (21). So the present formulation for the density at a nucleus is in accord with the previous applications of constraints. The difference lies mainly in the fact that the parameters $k$ and $k^{\prime}$ were determined definitely by the previous authors, while in our expression the constants $\alpha$ and $\beta$ are actually arbitrary.

Results for the density and its derivative at a nucleus for the neutral atoms from $\mathrm{He}$ and $\mathrm{Ar}$ are listed in Tables I and II, respectively, in which, for the purpose of comparison, exact results and Kato's cusp condition are also given. The calculations are performed using the Hartree-Fock densities of Clementi and Roetti [12]. $\nabla \rho$ and $\nabla^{2} \rho$ are computed analytically in terms of Slater-type orbitals. And the value of arbitrary constants $\alpha$ and $\beta$ we choose here is just the nuclear charge $Z$ of each atom. Excellent agreement between values from these expressions and exact values is observed. Accurate satisfaction of Kato's cusp condition between $\rho(0)$ and $\rho^{\prime}(0)$ is seen in the last column in Table II.

There have been recent works $[13,14]$ dealing with the electron density near or at nuclear cusps. An interesting formula which has been used is the Hiller-Sucher-Feinberg identity [15], which takes the form

$$
\rho(0)=\frac{1}{2 \pi}\left\langle\Psi\left|\frac{\partial v}{\partial r_{1}}-\frac{1}{r_{1}^{3}} \hat{x} \mathbf{L}_{1}^{2}\right| \Psi\right\rangle,
$$

where $r_{1}=\left|\mathbf{r}_{1}\right|, \hat{\mathbf{L}}_{1}=-i \mathbf{r}_{1} \times \nabla_{1}$ and $v=\Sigma_{j} v_{\text {ext }}\left(\mathbf{r}_{j}\right)+\Sigma_{j<k} 1 /$ $r_{j k}$, which is the summation of the electron-nuclear attractive and electron-electron repulsive potentials. This means that if one knows the wave function, and hence the density, of the system and its potentials, the density at nuclei cusps can be determined. The advantage of this approach lies in that it explicitly employs the potential of the system. On the other hand, however, one still has to use wave functions to compute $\rho(0)$ and, furthermore, calculations show that results are not good even when high-quality wave functions are employed. Some of the results listed in Tables I and II are from [13], obtained by using 6-311+ $+\mathrm{G}$ basis sets. Our approach is clearly superior. 
TABLE II. Density derivative at the nuclei cusps from Eqs. (17)-(20) for neutral atoms ranging from He to Ar. For comparison, values using the Hiller-Sucher-Feinberg identity and Kato's cusp condition are listed. The exact value of $\rho^{\prime}(0) / 2 Z \rho(0)$ is 1 .

\begin{tabular}{|c|c|c|c|c|c|c|}
\hline & & Density deriva & ve at nucleus ${ }^{\mathrm{a}}$ & & & Kato's cond. \\
\hline & Formula I & Formula II & Formula III & Formula IV & $\rho_{\mathrm{HSF}}^{\prime}(0)^{\mathrm{b}}$ & $-\rho^{\prime}(0) / 2 Z \rho(0)$ \\
\hline $\mathrm{He}$ & -14.420 & -14.420 & -14.420 & -14.420 & -12.744 & 1.002 \\
\hline $\mathrm{Li}$ & -83.479 & -83.479 & -83.479 & -83.479 & & 1.006 \\
\hline $\mathrm{Be}$ & -284.792 & -284.792 & -284.792 & -284.792 & -267.48 & 1.005 \\
\hline B & -722.601 & -722.601 & -722.601 & -722.601 & & 1.004 \\
\hline $\mathrm{C}$ & -1535.738 & -1535.738 & -1535.739 & -1535.738 & & 1.003 \\
\hline $\mathrm{N}$ & -2894.996 & -2894.996 & -2894.997 & -2894.996 & & 1.003 \\
\hline $\mathrm{O}$ & -5009.895 & -5009.894 & -5009.896 & -5009.896 & & 1.004 \\
\hline $\mathrm{F}$ & -8101.860 & -8101.858 & -8101.862 & -8101.860 & & 1.003 \\
\hline $\mathrm{Ne}$ & -12425.078 & -12425.076 & -12425.082 & -12425.078 & -11629 & 1.002 \\
\hline $\mathrm{Na}$ & -18364.846 & -18364.843 & -18364.852 & -18364.846 & & 1.001 \\
\hline $\mathrm{Mg}$ & -26274.491 & -26274.487 & -26274.501 & -26274.491 & & 1.001 \\
\hline $\mathrm{Al}$ & -36511.207 & -36511.200 & -36511.221 & -36511.207 & & 1.001 \\
\hline $\mathrm{Si}$ & -49489.964 & -49489.953 & -49489.985 & -49489.964 & & 1.001 \\
\hline $\mathrm{P}$ & -65638.236 & -65638.222 & -65638.266 & -65638.236 & & 1.001 \\
\hline$S$ & -85545.142 & -85545.121 & -85545.184 & -85545.142 & & 1.001 \\
\hline $\mathrm{Cl}$ & -109422.248 & -109422.219 & -109422.304 & -109422.248 & & 1.000 \\
\hline $\mathrm{Ar}$ & -138373.992 & -138373.954 & -138374.067 & -138373.992 & & 1.001 \\
\hline
\end{tabular}

${ }^{\mathrm{a}}$ Formulas I-IV represent Eqs. (17)-(20), respectively.

${ }^{\mathrm{b}}$ From Ref. [13].

\section{EXCHANGE-ONLY AND EXCHANGE-CORRELATION POTENTIALS AT A NUCLEAR CUSP}

Although various approximate forms of the exchangeonly and exchange-correlation energy functionals have been proposed in recent years, not until recently did we know the exchange-only potential and the exchange-correlation potential by numerical solution of the Kohn-Sham equation. Surprisingly it was not known until a very recent paper by Umrigar and Gonze [9] that, although approximate functionals for $E_{\mathrm{x}}[\rho]$ and $E_{\mathrm{xc}}[\rho]$ do yield improvements over the conventional LDA approach in energetics, most of these potentials are in poor agreement with the exact potentials both near or at nuclei cusps and in long-range regions. For example, as mentioned in Ref. [9], all the GGA approximate potentials, such as those of Langreth-Mehl [16], PerdewWang ' 86 [17], Perdew-Wang '91 [18], Becke '88 [19], and so on, diverge at nuclear cusps. Only the LDA potential remains finite. This awkward situation stimulates the search for new approximate functionals which behave properly both in cusp and in asymptotic regions. It also shows the need to find accurate data for these potentials at nuclear cusps, from which one may judge how well an approximate potential behaves at cusps.
Letting $V_{\mathrm{x}}$ or $V_{\mathrm{xc}}$ replace $F$ in Eqs. (3)-(6), one obtains expressions for $V_{\mathrm{x}}(0)$ and $V_{\mathrm{xc}}(0)$. For example, assuming spherical symmetry,

$$
\begin{aligned}
V_{\mathrm{xc}}(0) & =-\frac{1}{4 \pi} \int \frac{\nabla^{2} V_{\mathrm{xc}}(\mathbf{r})}{|\mathbf{r}|} d \mathbf{r} \\
& =-\int r\left[V_{\mathrm{xc}}^{\prime \prime}(r)+\frac{2}{r} V_{\mathrm{xc}}^{\prime}(r)\right] d r .
\end{aligned}
$$

By employing the numerical data for $V_{\mathrm{x}}(\mathbf{r})$ and $V_{\mathrm{xc}}(\mathbf{r})$ from the Nagy method [20] and the Zhao-Parr method [21], we have calculated $V_{\mathrm{x}}(0)$ and $V_{\mathrm{xc}}(0)$ for five neutral atoms, He, $\mathrm{Be}, \mathrm{Ne}, \mathrm{Mg}$, and $\mathrm{Ar}$, as shown in Tables III and IV, respectively. In the paper of Umrigar and Gonze [9], although the discrete values of $V_{\mathrm{x}}(0)$ and $V_{\mathrm{xc}}(0)$ of helium were not given, the authors did plot the curves of $V_{\mathrm{x}}(\mathbf{r})$ and $V_{\mathrm{xc}}(\mathbf{r})$, which show that the value of $V_{\mathrm{x}}(0)$ is around -1.7 , and $V_{\mathrm{xc}}(0)$ around -1.8 , in agreement with our results.

To check that these results at a nucleus are accurate, we note that the contributions to values of $V_{\mathrm{x}}(0)$ and $V_{\mathrm{xc}}(0)$ come from quite a long range. The results are relatively in-

TABLE III. Values of the exchange-only potential at the nuclear cusp $V_{\mathrm{x}}(0)$ for neutral atoms of $\mathrm{He}, \mathrm{Be}$, $\mathrm{Ne}$, and $\mathrm{Ar}$ (a.u.). Formulas I-IV denote Eqs. (3)-(6), all with $F=V_{\mathrm{x}}$.

\begin{tabular}{lcccccc}
\hline \hline Atom & I & II & III & IV & $-Z_{\text {eff }}$ & $-J_{1 s} / 2$ \\
\hline $\mathrm{He}$ & -1.708 & -1.697 & -1.704 & -1.703 & -1.668 & -1.688 \\
$\mathrm{Be}$ & -3.353 & -3.351 & -3.357 & -3.358 & -3.015 & -3.684 \\
$\mathrm{Ne}$ & -7.424 & -7.399 & -7.406 & -7.409 & -7.502 & -9.622 \\
$\mathrm{Ar}$ & -13.670 & -13.665 & -13.668 & -13.677 & -13.346 & -17.555 \\
\hline \hline
\end{tabular}


TABLE IV. Values of the exchange-correlation potential at the nuclear cusp $V_{\mathrm{xc}}(0)$ for a few neutral closed-shell atoms (a.u.). Formulas I-IV denote Eqs. (3)-(6), all with $F=V_{\mathrm{xc}}$.

\begin{tabular}{lccccc}
\hline \hline Atom & I & II & III & IV & Estimated $^{\text {a }}$ \\
\hline $\mathrm{He}$ & -1.815 & -1.755 & -1.815 & -1.817 & -2.29 \\
$\mathrm{Be}$ & -3.123 & -3.055 & -3.122 & -3.116 & -4.50 \\
$\mathrm{Ne}$ & -8.208 & -8.154 & -8.209 & -8.215 & -11.80 \\
$\mathrm{Mg}$ & -9.703 & -9.645 & -9.703 & -9.707 & \\
$\mathrm{Ar}$ & -17.587 & -17.505 & -17.581 & -17.567 & -22.10 \\
\hline \hline
\end{tabular}

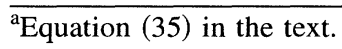

sensitive to the behavior of $\boldsymbol{\nabla} V_{\mathrm{x}}(\mathbf{r})$ or $\nabla^{2} V_{\mathrm{xc}}(\mathbf{r})$ near cusps, where errors are likely in numerical calculations. In Fig. 1, the integrand of Eq. (24) is plotted for the exchangecorrelation potential of helium.

\section{DISCUSSION}

It can be seen from Table III that the values of $V_{\mathrm{x}}(0)$ and $V_{\mathrm{xc}}(0)$ both are close to minus the nuclear charge $Z$ of the atom. This fact can serve as guidance in the finding of better approximate functionals for $E_{\mathrm{x}}[\rho]$ and $E_{\mathrm{xc}}[\rho]$.

To model this interesting behavior, it may be assumed that near the nuclear cusp the main component in the density and first-order density matrix is the $1 s$ orbital contribution [23]. One then has an approximate expression for $V_{\mathrm{x}}(0)$ :

$$
V_{\mathrm{x}}(0)=-J_{1 s} / 2,
$$

where $J_{1 s}$ is the electron-electron repulsion energy between $1 s$ orbitals. If one supposes that the density takes the form

$$
\rho^{1 s}(r)=A \exp \left(-2 Z_{\mathrm{eff}} r\right),
$$

and the first-order density matrix has the form

$$
\rho_{1}^{1 \mathrm{~s}}\left(r, r^{\prime}\right)=A \exp \left(-Z_{\mathrm{eff}} r-Z_{\mathrm{eff}} r^{\prime}\right),
$$

then one obtains the exchange energy functional

$$
E_{\mathrm{x}}[\rho]=\int \frac{2 \pi}{Z_{\mathrm{eff}}} \frac{\rho}{\ln (\rho / A)}\left[A-\rho+\frac{1}{2} \rho \ln (\rho / A)\right] d \mathbf{r} .
$$

The functional derivative of $E_{\mathrm{x}}[\rho]$ leads to the exchange potential, which turns out to be

$$
V_{\mathrm{x}}(r)=-\frac{1}{r}\left(1+\frac{1}{2 Z_{\mathrm{eff}} r}\right)\left[1-\left(1+2 Z_{\mathrm{eff}} r\right) e^{-2 Z_{\mathrm{eff}} r}\right]
$$

At $r=0$, we have $V_{\mathrm{x}}(0)=-Z_{\text {eff }}$. Values of $-J_{1 s} / 2$ and $-Z_{\text {eff }}$ for a few neutral atoms are also listed in Table III. $Z_{\text {eff }}$ was obtained by a least-square-fit process of Eq. (29) with the first five points of $V_{\mathrm{x}}(r)$ for each atom. It is found that they are good approximations for $V_{\mathrm{x}}(0)$.

Another way to account for this is from the Kohn-Sham equation, which reads

$$
\left[-\frac{1}{2} \nabla^{2}+V_{\mathrm{eff}}(r)\right] \varphi_{i}=\varepsilon_{i} \varphi_{i},
$$

where

$$
V_{\mathrm{eff}}(r)=V_{\mathrm{ext}}(r)+V_{J}(r)+V_{\mathrm{xc}}(r)
$$

Thus $V_{\mathrm{xc}}(r)$ can be expressed as

$$
V_{\mathrm{xc}}(r)=\varepsilon_{i}+\frac{1}{2 \varphi_{i}} \nabla^{2} \varphi_{i}-V_{\mathrm{ext}}(r)-V_{J}(r)
$$

Near the nuclear cusp, the most important term is the $1 s$ orbital; therefore one has approximately

$$
\varphi_{1 s} \approx\left(\frac{\rho}{2}\right)^{1 / 2}
$$

Inserting Eq. (33) into Eq. (32), we derive an approximation for $V_{\mathrm{xc}}(0)$ :

$$
\begin{aligned}
V_{\mathrm{xc}}(0) \approx & \varepsilon_{1 s}-\frac{1}{8}\left[\frac{\nabla \rho(0)}{\rho(0)}\right]^{2}+\frac{1}{4}\left(\frac{\nabla^{2} \rho}{\rho}\right)_{\rho=0}-V_{\mathrm{ext}}(r \rightarrow 0) \\
& -V_{J}(0)
\end{aligned}
$$

By using Kato's cusp condition and supposing $\rho(r)=A \exp (-2 Z r)$, one finally reaches

$$
V_{\mathrm{xc}}(0) \approx \varepsilon_{1 s}+\frac{1}{2} Z^{2}-\left\langle\frac{1}{r}\right\rangle
$$

Values from the above equation for a few atoms are given in the last column of Table IV. Fair agreement with the calculated $V_{\mathrm{xc}}(0)$ is found.

\section{CONCLUDING REMARKS}

In this paper, four rigorous expressions to obtain the value of any quantity at nuclear cusps have been given in terms of its gradients or Laplacians over the whole range of integration. Applications to the electron density and its derivative for neutral atoms ranging from $\mathrm{He}$ to $\mathrm{Ar}$, and to the exchange-only and exchange-correlation potentials of a few neutral atoms, have been carried out. Excellent agreement with reference values is observed. As a first-order approximation, we have found that the value of $V_{\mathrm{x}}(\mathbf{r})$ and $V_{\mathrm{xc}}(\mathbf{r})$ at a nuclear cusp is minus the nuclear charge $Z$ of the corresponding atom. 


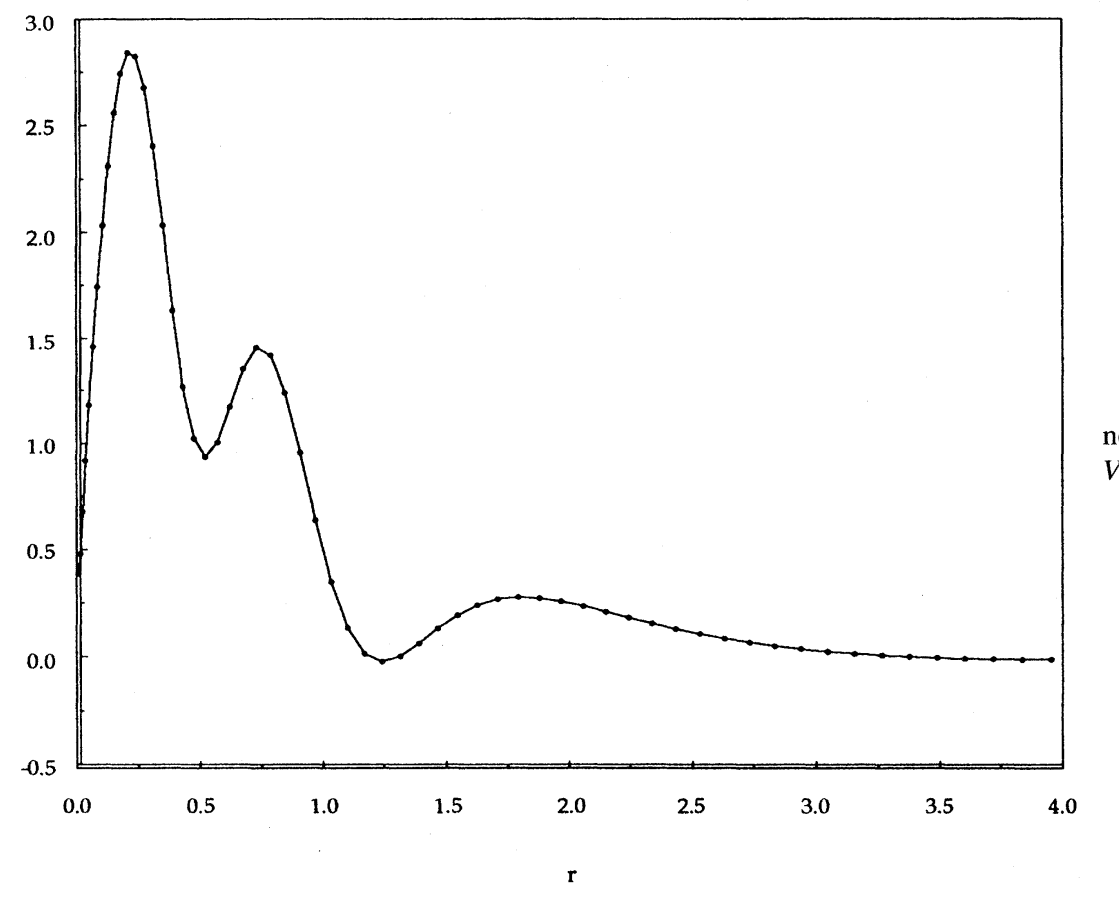

FIG. 1. Integrand of Eq. (24) for helium. The negative of the integral from zero to infinity is $V_{\mathrm{xc}}(0)$.

As a final remark, we would like to point out that, in terms of the gradient or Laplacian of any quantity, one actually can get the quantity itself anywhere in space from

$$
F(\mathbf{r})=-\frac{1}{4 \pi} \int \frac{\nabla^{2} F\left(\mathbf{r}^{\prime}\right)}{\left|\mathbf{r}-\mathbf{r}^{\prime}\right|} d \mathbf{r}^{\prime}
$$

or

$$
F(\mathbf{r})=\frac{1}{4 \pi} \int \frac{\left(\mathbf{r}-\mathbf{r}^{\prime}\right) \cdot \nabla^{\prime} F\left(\mathbf{r}^{\prime}\right)}{\left|\mathbf{r}-\mathbf{r}^{\prime}\right|^{3}} d \mathbf{r}^{\prime},
$$

or from other forms similar to Eqs. (5) and (6). The proof of this statement follows that given above, if one chooses $\psi=1 /\left|\mathbf{r}-\mathbf{r}^{\prime}\right|$ and $\phi=F\left(\mathbf{r}^{\prime}\right)$ in both identities of Green's theorem. Setting $F(\mathbf{r})=\rho(\mathbf{r})$, one obtains integro-differential equations for the density. Bader and Beddall [24] first gave Eq. (36) for the density.

\section{ACKNOWLEDGMENTS}

S.B.L. thanks Dr. Q. Zhao for help in the calculation of the exact exchange-only and exchange-correlation energy potentials using the program he provided. Discussion with Professor Robert C. Morrison is gratefully acknowledged. This research has been aided by grants from the National Science Foundation, the Petroleum Research Fund of the American Chemical Society, the Exxon Education Foundation, and the Hungarian-U.S. Science and Technology Joint Fund.
[1] For a review of DFT, see R. G. Parr and W. Yang, Density Functional Theory of Atoms and Molecules (Oxford University Press, Oxford, 1989); R. M. Dreizler and K. U. Gross, Density Functional Theory (Springer-Verlag, Berlin, 1990).

[2] W. Kohn and L. J. Sham, Phys. Rev. A 140, 1133 (1965).

[3] T. Kato, Commun. Pure Appl. Math. 10, 151 (1957).

[4] L. H. Thomas, Proc. Cambridge Philos. Soc. 23, 542 (1927).

[5] E. Fermi, Z. Phys. 48, 73 (1928).

[6] P. A. M. Dirac, Proc. Cambridge Philos. Soc. 26, 376 (1930).

[7] R. G. Parr and S. K. Ghosh, Proc. Natl. Acad. Sci. U.S.A. 83, 3577 (1986).

[8] See, for example, C. Lee, W. Yang, and R. G. Parr, Phys. Rev. B 37, 785 (1988).

[9] C. J. Umrigar and X. Gonze, Phys. Rev. A 50, 3827 (1994).
[10] J. D. Jackson, Classical Electrodynamics (Wiley, New York, 1975).

[11] Z. Zhou and R. G. Parr, Int. J. Quantum Chem. 42, 1759 (1992).

[12] E. Clementi and C. Roetti, Roothaan-Hartree-Fock Atomic Wavefunctions (Academic, New York, 1974).

[13] J. Cioslowski and M. Challacombe, Chem. Phys. Lett. 224, 175 (1994).

[14] A. Holas and N. H. March, J. Mol. Struct. (THEOCHEM) 315, 239 (1994).

[15] J. Hiller, J. Sucher, and G. Feinberg, Phys. Rev. A 18, 2399 (1978).

[16] D. C. Langreth and M. J. Mehl, Phys. Rev. B 28, 1809 (1983).

[17] J. P. Perdew and Y. Wang, Phys. Rev. B 33, 8800 (1986). 
[18] J. P. Perdew, in Electronic Structure of Solids '91, edited by P. Ziesche and H. Eschrig (Akademic Verlag, Berlin, 1991).

[19] A. P. Becke, Phys. Rev. A 38, 3098 (1988).

[20] A. Nagy, Philos. Mag. 69, 779 (1994).

[21] Q. Zhao and R. G. Parr, Phys. Rev. A 46, 2337 (1992); J. Chem. Phys. 98, 543 (1993).

[22] I. Porras and F. J. Galvez, Phys. Rev. A 46, 105 (1992).
[23] M. Berrondo, in Recent Progress in Many-Body Theories, edited by G. Zabolitzky, M. de Liano, M. Fortes, and J. W. Clark, Lecture Notes in Physics Vol. 142 (Springer, Berlin, 1981); M. Berrondo and O. Goscinski, Phys. Rev. 189, 10 (1969).

[24] R. F. W. Bader and P. M. Beddall, J. Chem. Phys. 56, 3320 (1972), footnote 10. 